\title{
A Estrutura Fatorial do Teste de Criatividade Figural Infantil ${ }^{1}$
}

\author{
Tatiana de Cássia Nakano ${ }^{2}$ \\ Pontificia Universidade Católica de Campinas \\ Ricardo Primi \\ Universidade São Francisco
}

\begin{abstract}
RESUMO - Com o objetivo de investigar evidências de validade do Teste de Criatividade Figural Infantil, instrumento que visa avaliar 12 características criativas (Fluência, Flexibilidade, Elaboração, Originalidade, Expressão de Emoção, Perspectiva Incomum, Perspectiva Interna, Fantasia, Movimento, Uso de Contexto, Extensão de Limites e Títulos Expressivos), uma análise fatorial foi realizada visando uma melhor compreensão acerca do agrupamento dessas características. Estudantes de $1^{\text {a a }} 8^{\text {a }}$ séries do Ensino Fundamental $(\mathrm{n}=1253,599 \mathrm{~F} / 654 \mathrm{M})$ responderam ao instrumento. A análise fatorial apontou uma estrutura composta por quatro fatores (enriquecimento de idéias, aspectos cognitivos, emotividade e preparação criativa), que foi adotado como modelo de interpretação do instrumento devido à sua consistência com a literatura científica.
\end{abstract}

Palavras-chave: criatividade; análise fatorial; desenhos; avaliação.

\section{The Factor Structure of the Test of Children's Figural Creativity}

\begin{abstract}
This paper aims to investigate the validity evidence of the Test of Figural Creativity for Children. Factor analysis was performed for a better understanding of the 12 characteristics of creativity assessed by the instrument (Fluency, Flexibility, Elaboration, Originality, Expression of Emotion, Uncommon Perspective, Internal Perspective, Fantasy, Movement, Use of Context, Extension of Boundaries and Expressive Titles). The instrument was administered to students from $1^{\text {st }}$ to $8^{\text {th }}$ grades of elementary school ( $\mathrm{n}=1253,599$ females and 654 males). Factor analysis indicated a structure consisting of four factors (cognitive aspects, enrichment of ideas, cognitive aspects, emotional and creative preparation) which was adopted as a model for interpretation of the instrument considering its consistency with the scientific literature.
\end{abstract}

Keywords: creativity; children; factor analysis; drawing; assessment.

Visando a ampliação do conceito de criatividade (compreendida como um dos componentes da inteligência) e a necessidade de avaliação dos diversos aspectos que compõem esta característica, Torrance (1966) desenvolveu uma bateria de testes para avaliar a criatividade através de aspectos cognitivos e emocionais nas dimensões verbal e figurativa, designada Torrance Tests of Creative Thinking - verbal and figural forms (Torrance \& Ball, 1978), que viria a se tornar um dos instrumentos mais reconhecidos internacionalmente na avaliação desse construto.

A importância desses testes refere-se ao fato de que, a partir da sua publicação em 1966, os mesmos tornaram-se os mais pesquisados e analisados que qualquer outro instrumento de avaliação da criatividade (Kim, 2006). Sua importância eve-se ao fato de seus estudos terem envolvido largas amostras e validações longitudinais, investigando a influência de iversas variaveis na expressao criativa, tais como gênero, raça e nível sócio-econômico, em individuos com diferentes linguagens e culturas. Tamanha a importância destes instrumentos que eles estão sendo utilizados amplamente

1 Apoio: FAPESP (Fundação de Amparo à Pesquisa do Estado de São Paulo) e CNPq.

2 Endereço para correspondência: PUC-Campinas - Programa de Pós-Graduação em Psicologia. Avenida Jonh Boyd Dunlop, s/n - Jardim Ipaussurama. Campinas, SP. CEP 13060-904. Fone: (19) 3343-6892. E-mail: tatiananakano@hotmail.com na identificação do indivíduo criativo, tendo sido traduzidos em mais de 30 diferentes idiomas e padronizados para outros países (Wechsler, 2004) e considerados apropriados para avaliação de indivíduos de diversas culturas, de acordo com Torrance e Ball (1990).

Os trabalhos de Torrance passaram a ser o ponto de partida para outros pesquisadores que viram na corrente psicométrica uma possibilidade de se tentar mensurar e classificar quantitativamente os níveis de criatividade dentro de determinado domínio. Isso porque, para construir a sua bateria, Torrance analisou os modos de pensamento de cientistas, artistas, escritores e outros grupos de profissionais que primam pela produção criativa. Depois, concebeu tarefas (testes) cuja resolução implicaria no recurso a processos cognitivos semelhantes aos que são usados por estas pessoas, de forma a avaliar as características de personalidade comuns a pessoas de grande destaque criativo na sociedade.

Torrance inicialmente fez em seus trabalhos a distinção entre quatro medidas de pensamento criativo (fluência, flexibilidade, elaboração e originalidade), baseado na teoria de Guilford (1950) e é com base nestes parâmetros que avaliou o desempenho dos sujeitos nos seus testes (Torrance \& Safter, 1999). Entretanto a partir de 1980, este autor passou a demonstrar a sua insatisfação com a limitação dos conceitos utilizados para avaliar a criatividade nos seus testes, uma vez que acabavam por reduzir a medida da criatividade à medida do pensamento divergente. A partir 
de um importante estudo longitudinal com intervalo de 22 anos entre as testagens, o autor decidiu publicar as mudanças que deveriam ser consideradas na avaliação do teste figurativo (Wechsler, 2004). A partir disso reelaborou sua avaliação, aumentando os indicadores da criatividade para quatorze, ao invés dos quatro propostos inicialmente, com a incorporação de novas categorias de análise e correção. Com isso deixam de ser avaliados somente os aspectos cognitivos da criatividade, passando a serem avaliados também os aspectos emocionais. Os novos indicadores propostos passam a ser: expressão de emoção, fantasia, movimento, combinação de idéias, extensão de limites, perspectiva incomum, perspectiva interna, uso de contexto, analogias e títulos expressivos, definidos em Torrance e Safter (1999) e explicitadas por Wechsler (2004).

Devido à popularidade do modelo de Torrance (1966) de avaliação da criatividade e ao grande número de características criativas avaliadas em seus testes, vários pesquisadores interessados em investigar as qualidades psicométricas desses instrumentais desenvolveram estudos com o objetivo de identificar sua estrutura fatorial com vistas a responder a uma das principais questões levantadas em relação aos instrumentos: qual a estrutura fatorial subjacente a essas características?. Neste sentido, relatos de dois tipos de análise fatorial foram encontrados na literatura: exploratória e confirmatória. $\mathrm{O}$ primeiro estudo exploratório a ser citado foi desenvolvido por Clapham (1998) considerando o fato de que um único fator parecia ser insuficiente para capturar a habilidade de pensamento divergente. Para isso investigou a estrutura dos testes figurais de Torrance formas A e B junto a 344 estudantes de Psicologia com idades entre 17 e 45 anos. Neste modelo as características criativas avaliadas eram fluência, originalidade, abstração dos títulos (chamado neste instrumento como títulos expressivos), elaboração e resistência ao fechamento prematuro (chamado de extensão de limites). Os resultados da análise dos componentes principais demonstrou que as características obtidas nas formas A e B refletem um único fator geral da criatividade, que explicaria 55,9\% e $50,3 \%$ da variância, respectivamente, indicando, de acordo com o autor, que a estrutura das duas formas poderia ser considerada equivalente.

Outro estudo da estrutura fatorial dos testes figural e verbal de Torrance foi realizado por Plass, Michael e Michael (1974) com 111 crianças de $6^{\mathrm{a}}$ série. Ambos instrumentos avaliam as características de fluência, flexibilidade, elaboração e originalidade, em nove atividades (seis verbais e três figurais), totalizando 30 itens. A análise fatorial exploratória por rotação varimax foi empregada buscando-se verificar quantas dimensões latentes não correlacionadas eram suficientes para explicar as correlações entre as variáveis. Os resultados indicaram a existência de sete fatores, mas se considerarmos somente os fatores pertencentes ao teste figural, foco do presente estudo, encontramos dois fatores que agruparam as tarefas (completar figuras e linhas) ao invés dos processos associados às variáveis (fluência flexibilidade, originalidade, etc.).

Heausler e Thompson (1988), por sua vez, utilizaram a forma A do teste figural de Torrance em 132 estudantes (69 da pré-escola e 63 da segunda série), avaliando cinco critérios (originalidade, elaboração, resistência do fechamento prematuro, fluência e abstração de títulos) e encontraram, após rotação varimax, uma matriz composta por dois fatores. $\mathrm{O}$ primeiro refletiria um fator criativo geral, que envolveria as características de originalidade, elaboração, resistência ao fechamento prematuro e fluência, enquanto que o segundo seria composto somente pela abstração de títulos.

Estudos envolvendo análise fatorial confirmatória foram desenvolvidos por Kim, Cramond e Bandalos (2006) junto a 3.000 estudantes (pré-escola, terceira série e sexta série) que, fazendo uso do Teste Figural forma A, testaram o modelo de um fator e um modelo de dois fatores. Os resultados indicaram níveis de ajuste muito melhores no modelo dos dois fatores, o primeiro fator chamado Inovativo, composto pelas características de fluência e originalidade e um segundo fator chamado Adaptativo, composto por elaboração e abstração de títulos, estando os dois fatores relacionados à característica de resistência ao fechamento prematuro.

Também fazendo uso da análise fatorial confirmatória, Kim (2006) conduziu um estudo com 500 estudantes de $6^{\mathrm{a}}$ série visando investigar se os testes de Torrance comportariam um ou dois fatores. Os achados apontaram que o modelo de dois fatores apresentou melhores resultados que o modelo de fator único. Os dois fatores encontrados mostraram-se consistentes com as descrições da teoria de adaptação-inovação de Kirton (1976), visto que o primeiro fator seria composto pelas características de fluência, originalidade e resistência ao fechamento prematuro, ao passo que o segundo fator seria composto por elaboração, abstração de títulos e resistência ao fechamento prematuro.

Desta forma, podemos verificar que desde que Guilford iniciou os estudos de criatividade propondo um modelo multidimensional (Chen \& Michael, 1993), muitos pesquisadores passaram a se embasar no modelo da criatividade como vários fatores independentes (Kim, Cramond \& Bandalos, 2006). Neste modelo a criatividade estaria localizada particularmente no modelo tridimensional de inteligência, com o processo de pensamento divergente sendo o mais importante. Ela poderia ser resultado da combinação dos produtos (seis) e do conteúdo (quatro), podendo ser encontrada de 24 modos diferentes, sendo que estas combinações de produto e conteúdo estariam relacionadas ao pensamento divergente (Sternberg \& Grigorenko, 2001).

Devido ao grande numero de fatores avaliados no modelo de Guilford (1960) e posteriormente no modelo de Torrance (1966), uma das maneiras mais utilizadas internacionalmente na condução dos estudos de validade desse tipo de instrumento tem sido a análise da estrutura latente de seus escores com o objetivo de identificar o tipo de validade de construto. Desde então vários instrumentos foram criados, derivados desses modelos e da adição de outras variáveis, de modo que vários estudos da estrutura fatorial foram publicados. Entretanto, conforme se observa, há uma grande variedade nos resultados. Parte dessa variabilidade pode ser atribuída às diferenças metodológicas. Os estudos variam no número e amplitude de testes utilizados, uso ou não dos escores divididos por tarefas ou somente variáveis, emprego da análise fatorial exploratória ou confirmatória, faixa etária e tamanho das amostras. Esta constatação aponta para o fato que, em parte, essas diferenças metodológicas podem estar na base das diferenças relatadas. 
Um problema metodológico importante ocorre ao se tentar detectar processos e ou traços psicológicos latentes por meio da análise da estrutura interna em tarefas de raciocínio divergente. Nesse contexto certas variáveis acabam sendo relativamente redundantes, como é o caso de fluência e flexibilidade já que a primeira é definida como o número de idéias apresentadas pelo sujeito a um dado estímulo e a segunda como o número de idéias diferentes. Ambas as variáveis tomam por base um único fato observado, número de idéias, sendo que, em uma delas (fluência) o importante é o número total, enquanto na outra (flexibilidade) o que vale é o número de idéias distintas. Assim poderíamos montar uma fórmula afirmando que fluência é igual ao número de idéias distintas (flexibilidade) mais o número de respostas repetidas (que são consideradas na avaliação da fluência mas não na da flexibilidade). Portanto flexibilidade estaria implicada na fluência em razão da sua definição, não sendo, de fato, dois indicadores distintos.

Preocupação similar foi relatada por Chase (1985) ao afirmar que os coeficientes de correlação entre Fluência, Flexibilidade e Originalidade são altos (entre 0,74 e 0,80), de forma que um simples escore poderia ser apropriado para representar os três subescores. Outros pesquisadores, como Heausler e Thomson (1988), Runco e Mraz (1992), Clapham (1998), também tem defendido a idéia de que as medidas do teste de Torrance não são independentes devido às altas correlações entre elas, tendo sido mais comum encontrar na literatura descrições acerca da alta correlação entre Fluência e Originalidade. Ainda de acordo com Heausler e Thomson (1988) desde 1972 Thorndike já chamava a atenção para a pequena evidência de consistência acerca das diferenças entre as medidas de fluência, flexibilidade e originalidade, não sendo surpresa, para o autor, que estes escores tendam a ser altamente correlacionados. Outros autores como Moraes e Azevedo (2009), Chase (1985) já teriam chamado atenção para o fato de que, durante a avaliação da criatividade, correlações demasiado elevadas aparecem entre diferentes parâmetros deste tipo de testes, nomeadamente entre a fluência, originalidade e flexibilidade, já que a primeira acaba por abarcar as outras, o que viria a exigir atenção e controle.

Visto que "diversos estudos tem sugerido diferentes preditores e número de fatores" nos testes de Torrance (Kim, 2006, p.257) e considerando que o processo de criação do Teste de Criatividade Figural Infantil (TCFI) baseou-se no teste figural de Torrance, no presente estudo as características avaliadas pelo instrumento infantil foram investigadas com o objetivo de identificar sua estrutura latente na tentativa de se compreender melhor a função cognitiva da criatividade. Os estudos fatoriais tem por objetivo descobrir as variáveis subjacentes (latentes) a partir da observação do agrupamento de informações em indicadores distintos. A hipótese implícita nos estudos que empregam a análise fatorial é que se as variáveis observadas são distintos efeitos de uma variável latente, então estarão correlacionados, de forma que a correlação entre indicadores distintos é uma evidência da existência daquele construto que seria, em certo sentido, a causa latente desses indicadores. No caso da criatividade vimos que algumas variáveis não são totalmente distintas, devido principalmente ao fato de estarem baseadas em um mesmo observável (respostas), o que pode fazer com que provavelmente apresentem uma alta correlação devido a um artefato metodológico, de forma a confundir a estrutura de covariância que é analisada em busca dos construtos. Desta forma, nesse tipo de estudo algum controle estatístico precisaria ser feito antes de submeter as matrizes à análise fatorial.

Ao mesmo tempo um outro cuidado deveria ser o de considerar a mesma variável nas diferentes tarefas que compõem o instrumento, de tal forma a se ter variáveis realmente distintas (flexibilidade, originalidade, por exemplo) medidas mais de uma vez, por exemplo, em diferentes tarefas. A partir desses cuidados metodológicos tornar-se-ia possível ter mais clareza acerca da estrutura fatorial dos processos psicológicos que explicam as variáveis do teste. É interessante notar que a maioria dos estudos encontrados na literatura realiza a análise das variáveis de uma forma geral (sem dividi-las por tarefa), além de não controlar a dependência linear de algumas variáveis. A única exceção foi o estudo de Plass, Michael e Michael (1974) que acabou por encontrar dados que apontaram que os fatores acabaram sendo compostos pelas tarefas (atividades) ao invés das variáveis. Entretanto devemos salientar que esse só controlou uma das variáveis apontadas mas não controlou a redundância entre as variáveis, de forma que se torna evidente que as características de fluência e flexibilidade em uma mesma tarefa estarão altamente correlacionadas, forçando a existência de um fator.

Diante disso apresentamos este estudo que buscou investigar a estrutura interna de um teste de criatividade figural, derivado do Teste de Pensamento Criativo de Torrance (Torrance, 1966), buscando investigar a questão: quais processos psicológicos estão na base das variáveis medidas?. Sua importância se refere ao fato de que nesse foi empregado um controle das colinearidades das variáveis, efetuando-se a análise das características criativas divididas por tarefa. Assim espera-se que esses ajustes ajudem a revelar com mais clareza a estrutura latente das variáveis em testes de pensamento criativo, tipo os testes de Torrance.

\section{Método}

\section{Participantes}

Utilizou-se uma amostra de conveniência, composta pelos participantes da pesquisa de normatização do instrumento. Considerou-se os resultados de 1.253 participantes, sendo 599 do sexo feminino e 654 do masculino, com idades entre 6 e 16 anos (média de 10,78 anos, DP=2,27), estudantes de $1^{\mathrm{a}}$ a $8^{\mathrm{a}}$ séries do Ensino Fundamental, 642 provenientes de escola particular e 611 de escola pública, de três cidades brasileiras, uma localizada na região sudeste, uma na região nordeste e outra no centro-oeste, conforme pode ser visualizado na Tabela 1.

\section{Instrumento}

O Teste Brasileiro de Criatividade Figural é uma medida desenvolvida por Nakano, Wechsler e Primi (2011) 
Tabela 1. Descrição da amostra

\begin{tabular}{ccccccccc}
\hline & $1^{\mathrm{a}}$ série & $2^{\mathrm{a}}$ série & $3^{\mathrm{a}}$ série & $4^{\mathrm{a}}$ série & $5^{\mathrm{a}}$ série & $6^{\mathrm{a}}$ série & $7^{\mathrm{a}}$ série & $8^{\mathrm{a}}$ série \\
\hline Escola Pública & 38 & 76 & 88 & 69 & 86 & 89 & 78 & 87 \\
Escola Particular & 69 & 67 & 67 & 101 & 100 & 79 & 84 & 75 \\
Sexo feminino & 52 & 65 & 79 & 72 & 84 & 89 & 81 & 77 \\
Sexo Masculino & 55 & 78 & 76 & 98 & 102 & 79 & 81 & 85 \\
Região Sudeste & 27 & 53 & 59 & 50 & 62 & 64 & 33 & 51 \\
Região Nordeste & 40 & 58 & 54 & 84 & 82 & 59 & 83 & 65 \\
Região Centro-Oeste & 40 & 32 & 42 & 36 & 42 & 45 & 46 & 46 \\
Total & 107 & 143 & 155 & 170 & 186 & 168 & 162 & 162 \\
\hline
\end{tabular}

baseada no Teste de Pensamento Criativo Figural de Torrance (Torrance, 1966; Torrance \& Ball, 1978, 1990). O instrumento é composto por três atividades, sendo pedida na primeira atividade a elaboração de um desenho a partir de um estímulo pouco definido (fazer um desenho), na segunda atividade deve-se completar o desenho a partir de 10 estímulos incompletos (acabar um desenho) e na terceira atividade fazer o maior número de desenhos a partir do mesmo estímulo repetido 30 vezes (fazer desenhos a partir de um semiquadrado), de forma que o instrumento permite ao sujeito a elaboração de até 41 respostas sob a forma de desenhos, considerando-se as três atividades. As características avaliadas pelo instrumento são 12: Fluência (número de idéias relevantes oferecidas pelo sujeito), Flexibilidade (diversidade de tipos ou categorias de idéias), Elaboração (adição de detalhes ao desenho básico), Originalidade (idéias incomuns), Expressão de Emoção (expressão de sentimentos, tanto nos desenhos quanto nos títulos), Fantasia (presença de seres imaginários, de contos de fada ou ficção científica), Movimento (clara expressão de movimento nos desenhos ou títulos), Perspectiva Incomum (pessoas ou objetos desenhados sobre ângulos não usuais), Perspectiva Interna (visão interna de objetos ou parte do corpo das pessoas, sob a forma de transparência), Uso de Contexto (criação de um ambiente para o desenho), Extensão de Limites (estender os estímulos antes de concluir os desenhos), Títulos Expressivos (ir além da descrição óbvia do desenho, abstraindo-o).

Os testes foram corrigidos por um dos pesquisadores, experiente na correção da versão brasileira dos Testes de Torrance. As características são corrigidas de acordo com a sua ocorrência em cada um dos estímulos e em cada atividade, dando origem a pontuações brutas em cada processo (exemplo: Elaboração na atividade 3). No modelo do instrumento são oferecidas tabelas de normas divididas por série, de forma que os indivíduos são comparados com iguais, cuidado necessário frente à amplitude de séries envolvidas (consequentemente, de idades).

Os estudos iniciais de validade e precisão do Teste de Criatividade Figural Infantil realizadas por Nakano (2003) indicaram valores entre 0,81 e 0,94 de correlação para validade concorrente com o Teste Figural de Torrance e índices entre 0,84 e 0,95 de correlação para a precisão por meio do teste e reteste, configurando-se em bons indicadores de precisão e validade em amostras brasileiras (Nakano \& Wechsler, 2006a). Os resultados demonstraram ainda que o instrumento sofreu influência significativa das variáveis sexo e série (Nakano \& Wechsler, 2006b).

\section{Procedimento}

O instrumento foi aplicado por um dos pesquisadores, de forma coletiva em sala de aula, após obtenção da autorização do Comitê de Ética em Pesquisa e assinatura, pelos pais dos alunos, do termo de consentimento livre e esclarecido. Considerando que o TCFI visa avaliar 12 características criativas, nas três atividades do teste, com algumas exceções (Fluência e Flexibilidade na atividade 1 que não são computadas devido ao fato da atividade permitir a elaboração de somente uma resposta e Extensão de Limites que só é avaliada na atividade 3), o número total de subescores considerados na análise fatorial foi 32 .

Entretanto, antes da realização da Análise Fatorial algumas medidas foram tomadas visando a realização de correções estatísticas com o objetivo de se chegar a uma matriz de correlação menos suscetível ao efeito dos artefatos metodológicos, como apontado pela literatura. Conforme apresentado anteriormente, alguns itens apresentam uma colinearidade decorrente da forma como são pontuadas (especialmente fluência, flexibilidade e originalidade). Ao analisar os itens do teste observou-se que neste estudo a fluência e flexibilidade foram consideradas potencialmente as mais problemáticas uma vez que a correlação encontrada entre as duas foi de moderada para alta $(r=0,54$ na Atividade 2 e $r$ $=0,85$ na Atividade $3, p<0,01)$. Como já foi mencionado, ao analisar suas definições pode-se perceber que a avaliação das mesmas se mostrava de certa forma redundante visto que para que exista a flexibilidade (diversidade) há de haver necessariamente a fluência (respostas adequadas), de forma que foi elaborada a hipótese de que a flexibilidade seria, de certa forma, uma fluência mais elaborada. O problema é que parte dessa associação está relacionada à maneira como estas são definidas e não em razão de um processo psicológico comum que se quer investigar. Assim, a solução proposta como forma de controlar essa redundância foi o emprego da regressão linear com a finalidade de prever a fluência a partir da flexibilidade. 


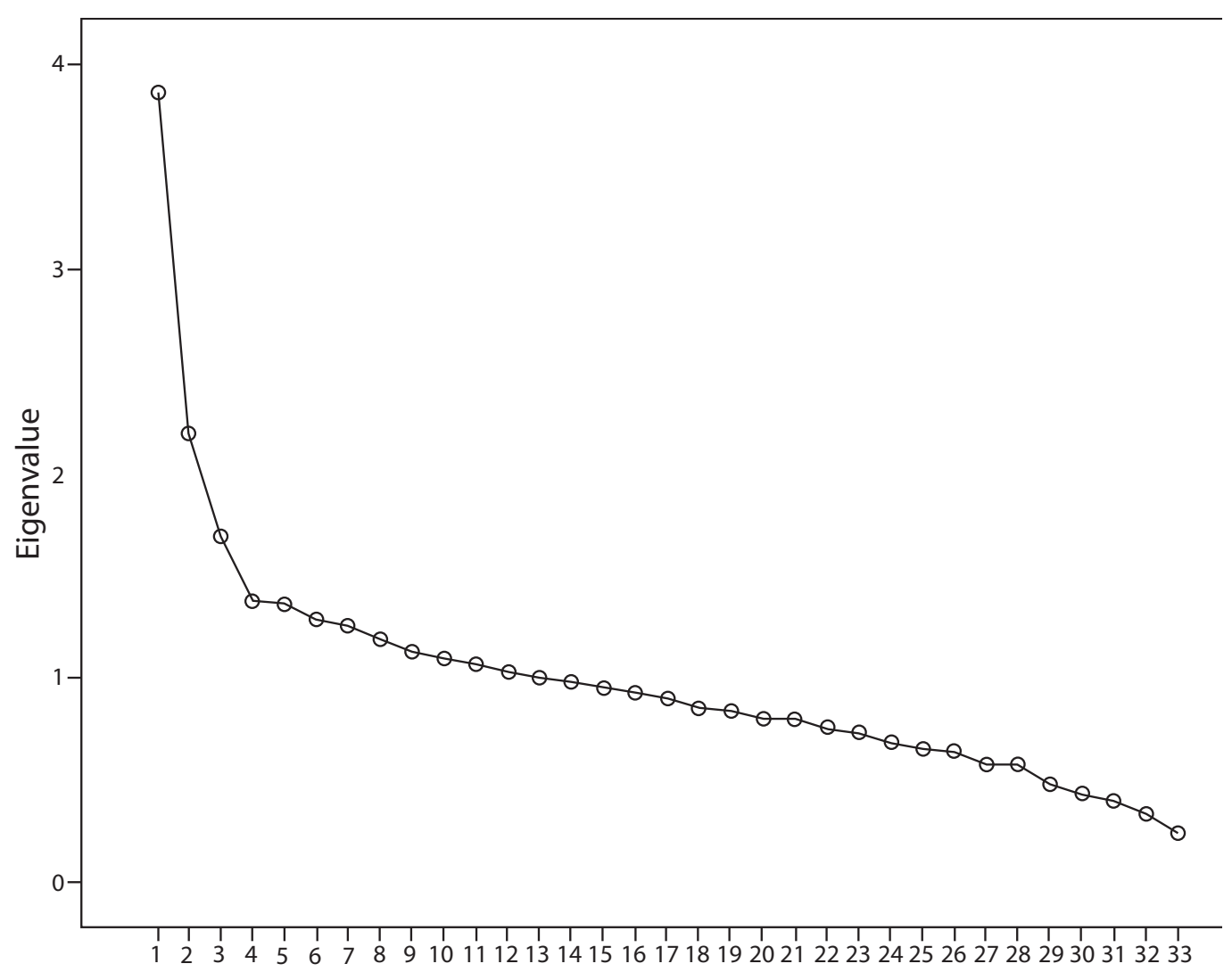

Figura 1. Scree plot resultante da análise fatorial do TCFI

Os resíduos padronizados dessa análise representam a parcela de fluência não associada à flexibilidade (repetições e/ou produtividade de idéias nas mesmas categorias). Assim na análise fatorial ao invés de se considerar o escore em fluência optou-se por considerar os resíduos mencionados acima. Isto porque, se houvesse a simples inserção da fluência, ao invés do resíduo, teríamos duas vezes o mesmo componente (número de idéias diferentes), uma vez que a fluência é definida como o número de idéias diferentes + número de repetições e a flexibilidade como o número de idéias diferentes, aumentando-se artificialmente as correlações por atividade. Ao se considerar somente o resíduo teremos dois componentes distintos separados (idéias diferentes e repetições). A partir dessa decisão foi realizada a análise fatorial das variáveis divididas por atividade. A expectativa foi que, efetuando a correção estatística da colinearidade e analisando-se escores por atividade, teríamos uma matriz mais adequada para se tentar evidenciar fatores ligados aos processos psicológicos subjacentes.

\section{Resultados e Discussão}

O objetivo desse estudo foi investigar a estrutura interna do TCFI buscando-se evidenciar a validade desse instrumento de forma a compreender melhor os construtos que seus itens representam. Para isso a matriz de correlação entre as variáveis foi submetida à análise fatorial exploratória. Inicialmente procedeu-se a checagem de adequação da matriz de correlação para a análise fatorial (Marroco, 2003). O teste de esfericidade de Bartlett, testando-se a hipótese nula de que a matriz de correlação era igual à matriz identidade, apontou um resultado altamente significativo $\left(\chi^{2}=6830,5\right.$; $N=496 ; p<0,001$ ), de forma que a hipótese nula pode ser descartada. A análise de adequação da amostra utilizando o método KMO (Kaiser-Meyer-Olkim), que testa se as correlações parciais entre as variáveis são baixas, indicando, portanto, que as correlações sugerem maior interconexão entre elas, também foi realizada e resultou em um valor adequado $(0,71)$, atestando a adequação dos dados para a realização da análise fatorial.

Inicialmente uma análise fatorial por componentes principais com rotação oblimin foi efetuada e mostrou que as correlações entre os fatores eram, em sua maioria, próximas de zero. Sendo assim, optou-se pela aplicação da rotação varimax, que se adequou aos dados e é mais parcimoniosa (Tabachnick, \& Fidell, 1996). Na primeira solução foram encontrados 12 fatores com eigenvalues superiores a um, contudo, é sabido que esse procedimento não é o mais adequado como critério de extração de fatores então a decisão do número de fatores se baseou em dois procedimentos mais adequados (Reise, Waller \& Comrey 2000): o primeiro foi a análise do scree-plot (Figura 1), seguindo os procedimentos orientados por Carroll (1985) e Field (2009) que sugeriu a retenção de quatro fatores. O segundo critério foi derivado da análise paralela (Watkis, 2006) que gera um conjunto de matrizes correlações casuais com o mesmo número de variáveis e sujeitos da pesquisa e se extrai os componentes principais computando-se a média dos eigenvalues. Com base nessas simulações o critério é que os fatores retidos de- 
vam ter eigenvalues maiores que os eigenvalues produzidos na simulação com dados randômicos. Os resultados dessa análise mostraram-se coerentes com aqueles apresentados pelo scree-plot ao sugerirem a retenção de quatro fatores. Os eigenvalues inicias extraídos foram 4,01, 2,35, 1,83, 1,41 (sendo o quinto 1,35). A análise paralela produzindo 100 matrizes de correlações randômicas de 32 variáveis e 1253 sujeitos (empregando-se uma sintaxe para o SPSS, Enzmann, 1997) sugere que se extraiam fatores com eigenvalues maiores que 1,35.

Com base nesses critérios optou-se pela aplicação de uma nova análise fatorial, impondo-se a extração de quatro fatores que resultou nos eigenvalues (após a rotação ortogonal) de 2,$77 ; 2,55 ; 2,46$ e 1,83 que explicariam, 8,67\%; $7,96 \% ; 7,70 \%$ e $5,72 \%$ (somando $30,0 \%$ ) da variância total. Para a definição dos fatores foram analisadas as variáveis cujas cargas fossem iguais ou superiores a 0,20. Embora esse critério seja mais leniente que o habitualmente recomendado de 0,32 ele permite ser mais inclusivo, fato que é condizente com a atitude exploratória desse estudo. O objetivo nesse momento da análise foi garantir uma certa abrangência a fim de se entender melhor o construto que cada fator representa. Assim todas as variáveis correlacionadas, ainda que em baixa magnitude, foram consideradas. Importante salientar que essa decisão baseou-se no modelo proposto por Stevens (1992), segundo o qual, para amostras maiores que 600, uma carga maior de 0,21 pode ser considerada significativa, a nível de 0,01 (bilateral). Assim na Tabela 2 são apresentados os fatores extraídos, seus conteúdos, carga fatorial e estatística descritiva de cada uma das características (pontuação mínima, máxima, média e desvio padrão). Nela, uma observação refere-se às características de Fluência 2 e Fluência 3, cujos valores, devido ao controle de colinearidade realizado, foram estimadas a partir da fluência residual, anteriormente explicada, de modo que seus valores mostram-se diferentes dos demais visto que os mesmos encontram-se padronizadas em medida $\mathrm{z}$.

As características criativas ao se agruparem em 4 fatores revelaram congruência do teste e o construto da criatividade dentro do modelo que ele se propõe a medir. Com a finalidade de que as soluções fatoriais extraídas confirmassem a pertinência dos fatores encontrados (em termos de conteúdo) e as características psicométricas das dimensões identificadas, considerando-se que cada uma das medidas avaliadas pelo instrumento representa um tipo de característica que geralmente é apresentada pelas pessoas criativas, aquelas características que obtiveram carga em mais de um fator acabaram por ser mantidas em somente um deles, geralmente optando-se por aquele na qual ela apresentou maior carga, mas em alguns casos, por exemplo, naqueles em que há carga maior em um fator mas negativa, optou-se por mantê-la no fator em que a carga fosse positiva ou naquele em que o conteúdo fosse mais pertinente com a literatura.

O primeiro fator foi chamado de Enriquecimento de Idéias, devido às características que o compõem, que envolvem ver a situação de uma forma mais detalhada, com acréscimo de detalhes e o enriquecimento da resposta, visualizada em um contexto mais amplo e dinâmico e ponto de vista diferente. É composto pelas características Elaboração (nas atividades 1, 2 e 3), Uso de Contexto (nas atividades 2 e 3), Perspectiva Interna (atividades 2 e 3), Perspectiva Incomum (atividades 1, 2 e 3), Movimento (atividades 2 e 3 ).

O segundo fator, chamado de Emotividade foi composto por características que envolvem o uso de recursos criativos ligados à uma percepção mais emocional, facilitadora no processo de descoberta de uma nova idéia. É composta pelas características de Expressão de Emoção (atividades 2 e 3), Títulos Expressivos (atividades 1, 2 e 3) e Fantasia (atividades 2 e 3). Importante salientar que a característica Títulos Expressivos embora também contemple a expressão de recursos cognitivos, é considerada, dentro dos modelos que embasam este instrumento (Torrance \& Ball, 1978, 1990; Wechsler, 2004; Torrance \& Safter, 1999), como uma característica criativa emocional.

O terceiro fator foi chamado de Aspectos Cognitivos, visto que é composto por características criativas que fazem uso de recursos cognitivos, que envolvem a busca de soluções diferenciadas, originais e que vão além dos limites estabelecidos. É composto pelas características de Fluência residual (nas atividades 2 e 3), Flexibilidade (nas atividades 2 e 3), Originalidade (nas atividades 2 e 3 ) e Extensão de Limites (na atividade 3). A existência de um fator cognitivo concorda com a proposta de Guilford (1960) e posteriormente com o modelo inicial de Torrance (1966).

Por fim o quarto fator foi chamado de Preparação Criativa, o qual agrupo somente características pertencentes à primeira atividade do teste (Elaboração, Originalidade, Uso de Contexto, Movimento e Perspectiva Interna), salientando-se o fato da sua proposta ser bem diferente das outras duas atividades, solicitando-se a realização de um único desenho. Por este motivo foi considerado um fator de "esquentamento", uma oportunidade de treino para a realização das atividades posteriores que permitem o uso mais livre da criatividade e um maior número de respostas. Enquanto as atividades 2 e 3 do teste propõem a realização de desenhos a partir de vários estímulos, a atividade 1 é constituída somente por um desenho, o que acaba limitando a amplitude de pontuação além de não ser possível pontuar Fluência e Flexibilidade. É sabido o efeito redutor que a restrição da amplitude tem nos coeficientes de correlação (Goodwin \& Leech, 2006). Assim a diminuição da amplitude e representatividade das variáveis provavelmente pode ter tido um impacto, de forma a reduzir as correlações entre as variáveis dessa atividade com as outras. Outras investigações acerca desse fator são recomendadas com a finalidade de se investigar a sua constituição uma vez que quatro características carregaram positivamente e duas negativamente, inclusive com uma delas (Elaboração) obtendo carga alta em dois fatores.

Após a realização da Análise Fatorial pudemos verificar que o modelo de quatro fatores confirma a literatura científica ao apresentar uma separação entre as características criativas cognitivas e características criativas emocionais descritoras da pessoa criativa. Convém, no entanto, chamar a atenção para o fato de que no modelo multidimensional do construto outras facetas devem ser consideradas, tais como a influência dos fatores ambientais que também podem impactar de forma significativa a produção criativa, visto que a presente pesquisa focou-se exclusivamente na investigação das características da pessoa criativa. Atentou-se ainda para 
Tabela 2. Estatística descritiva e cargas fatoriais, consistência interna e comunalidades dos fatores do Teste de Criatividade Figural Infantil

\begin{tabular}{|c|c|c|c|c|c|c|c|c|c|}
\hline & \multicolumn{4}{|c|}{ Fatores } & & \multirow[t]{2}{*}{ Mínimo } & \multirow[t]{2}{*}{ Máximo } & \multirow[t]{2}{*}{ Média } & \multirow[t]{2}{*}{ D.P. } \\
\hline & 1 & 2 & 3 & 4 & $h^{2}$ & & & & \\
\hline Alfa & 0,63 & 0,65 & 0,59 & 0,5 & & & & & \\
\hline elab2 & 0,773 & & & & 0,649 & 0 & 156 & 14,94 & 9,78 \\
\hline elab3 & 0,651 & & & & 0,632 & 0 & 64 & 18,71 & 10,11 \\
\hline ucont2 & 0,55 & & & & 0,38 & 0 & 10 & 0,91 & 1,49 \\
\hline pint2 & 0,346 & & & & 0,147 & 0 & 4 & 0,34 & 0,6 \\
\hline pint3 & 0,333 & & & & 0,161 & 0 & 6 & 0,78 & 1 \\
\hline mov2 & 0,323 & & & & 0,147 & 0 & 5 & 0,45 & 0,75 \\
\hline pinc2 & 0,295 & & & & 0,106 & 0 & 4 & 0,35 & 0,66 \\
\hline mov3 & 0,274 & & & & 0,086 & 0 & 5 & 0,24 & 0,54 \\
\hline ucont3 & 0,231 & & & & 0,062 & 0 & 13 & 0,12 & 0,54 \\
\hline fant1 & & & & & 0,022 & 0 & 1 & 0,03 & 0,16 \\
\hline tit3 & & 0,816 & & & 0,673 & 0 & 22 & 0,74 & 2,14 \\
\hline tit2 & & 0,742 & & & 0,612 & 0 & 20 & 1,31 & 2,57 \\
\hline emo3 & & 0,652 & & & 0,439 & 0 & 4 & 0,07 & 0,37 \\
\hline emo2 & & 0,562 & & & 0,349 & 0 & 6 & 0,18 & 0,57 \\
\hline fant3 & & 0,379 & & & 0,17 & 0 & 3 & 0,1 & 0,35 \\
\hline tit1 & & 0,377 & & & 0,227 & 0 & 2 & 0,47 & 0,56 \\
\hline fant2 & & 0,272 & & & 0,144 & 0 & 5 & 0,22 & 0,54 \\
\hline emo1 & & & & & 0,049 & 0 & 2 & 0,08 & 0,3 \\
\hline orig3 & & & 0,727 & & 0,575 & 0 & 23 & 2,6 & 2,34 \\
\hline flex3 & & & 0,706 & & 0,613 & 0 & 28 & 10,62 & 4,76 \\
\hline extlim3 & & & 0,597 & & 0,373 & 0 & 30 & 3,06 & 3,19 \\
\hline flex2 & & & 0,455 & & 0,228 & 0 & 10 & 7,15 & 1,57 \\
\hline orig2 & & & 0,423 & & 0,198 & 0 & 9 & 2,52 & 1,73 \\
\hline flu3 & & & 0,365 & & 0,168 & $-1,2$ & 8,16 & 0 & 1 \\
\hline pinc3 & 0,34 & & & & 0,267 & 0 & 9 & 0,84 & 1,15 \\
\hline flu2 & & & 0,283 & & 0,118 & $-6,24$ & 3,57 & 0 & 1 \\
\hline ucont1 & & & & 0,67 & 0,479 & 0 & 1 & 0,48 & 0,5 \\
\hline elab1 & 0,423 & & & 0,552 & 0,488 & 0 & 22 & 6,13 & 3,78 \\
\hline orig1 & & & & $-0,541$ & 0,338 & 0 & 1 & 0,31 & 0,46 \\
\hline pint1 & & & & 0,482 & 0,237 & 0 & 2 & 0,22 & 0,43 \\
\hline mov1 & & & & 0,436 & 0,205 & 0 & 6 & 0,17 & 0,45 \\
\hline pinc1 & 0,311 & & & $-0,422$ & 0,277 & 0 & 2 & 0,12 & 0,32 \\
\hline
\end{tabular}

o fato que somente duas medidas não obtiveram carga em nenhum fator (Expressão de Emoção e Fantasia, ambas na atividade 1) devendo ser investigadas, posteriormente, de forma mais aprofundada.

Por fim realizou-se o levantamento da correlação entre os fatores com a finalidade de verificar a associação entre eles. Os resultados mostram a existência de correlação significativa entre todos os fatores (com intervalo de confiança de $99 \%$ ) com valores entre $r=0,527$ e r $=0,084$ : fator $1 \mathrm{x}$ fator
$2=0,334$; fator $1 \times$ fator $3=0,527$; fator $1 \times$ fator $4=0,375$; fator $2 \mathrm{x}$ fator $3=0,163$; fator $2 \mathrm{x}$ fator $4=0,084$; fator $3 \mathrm{x}$ fator $4=0,128)$. Estes resultados permitem afirmar que os fatores possuem partes compartilhadas (variância comum), resultado esperado devido ao fato de terem sido pontuados a partir da análise das mesmas respostas (desenhos), mas também apontaram a existência de partes específicas (variância única), que justificam a manutenção do modelo. Em relação às altas correlações entre as variáveis, Kim (2006) confirma 
esta percepção ao afirmar que "elas podem ser em partes resultado do fato dos diferentes subescores serem baseados no mesmo estímulo" (p.256).

Dois importantes diferenciais podem ser apresentados em relação à forma como este estudo foi conduzido. Enquanto todas as pesquisas que realizaram a análise fatorial das características avaliadas nos Testes de Torrance sempre a fizeram considerando-se as características criativas de uma forma geral, o presente estudo trabalhou com as variáveis por atividade para diversificar a observação de manifestações distintas, isto é, aumentar a observação de indicadores supostamente ligados a um construto comum.

O segundo ponto relaciona-se ao controle da colinearidade das variáveis, como forma de tentar melhorar a qualidade dos indicadores, através da remoção de pseudocorrelações, que eventualmente poderiam confundir a estrutura de covariância entre eles, base das inferências dos construtos que são hipotetizados como causa das variáveis. É interessante notar que, conforme hipotetizado, com exceção do Fator 4, os fatores não estão definidos por variáveis de uma só tarefa (atividade) como foi encontrado por Plass, Michael e Michael (1974). Ao contrário, são definidos por variáveis que representam um construto comum (agrupando-se as características criativas e não as atividades do teste). De fato se fosse feita uma análise dessa mesma matriz sem o controle da colinearidade de fluência e flexibilidade, os fatores ficariam muito mais associados às tarefas do que se encontrou na presente análise, decisão que previamente eliminou a redundância entre elas. Isso reforça a hipótese de que as diferenças de resultados entre os estudos relacionam-se mais com as decisões metodológicas tomadas.

Em resumo, foram encontrados quatro fatores, diferentemente do fator único ou modelo de dois fatores que tem sido relatado na maior parte dos estudos encontrados na literatura. Entretanto convém salientar que, o modelo de quatro fatores fez sentido perante a teoria que embasa o instrumento visto que, diferenciou principalmente os aspectos cognitivos e emocionais da criatividade. Além disso, o presente estudo efetuou uma série ajustes metodológicos cuidadosos para se produzir uma matriz de indicadores mais adequada para a análise fatorial. A prova disso é que quando esses controles são removidos os resultados ficam mais condizentes com a teoria que embasa o instrumento.

Convém atentar para o fato que, embora a maior parte dos estudos encontrados aponta uma solução composta por dois fatores (Heausler \& Thompson, 1988; Plass, Michael \& Michael, 1974; Kim, Cramond \& Bandalos, 2006; Kim, 2006), estas análises foram conduzidas considerando-se quatro ou cinco características criativas (fluência, flexibilidade, elaboração, originalidade e resistência ao fechamento prematuro), avaliadas de uma forma geral, sem levar em conta a diferenciação da sua ocorrência de acordo com a atividade, decisão que traz como resultado o agrupamento em dois fatores. Neste estudo, além de se considerar um número bem maior de características $(\mathrm{n}=12)$, a atividade em que ela ocorre também foi considerada devido à diferença entre os processos associados a cada tarefa de forma que, ao se trabalhar com 32 processos diferentes, encontrou-se um modelo de quatro fatores que foi adotado para o instrumento.
Por fim chama-se a atenção para a constatação de que as altas correlações entre os fatores demonstrou que estes possuem partes compartilhadas (variância comum), de forma que apesar do modelo ter apontado quatro fatores, a correlação entre eles permite que se afirme a existência de um fator mais amplo de segunda ordem, semelhante a um fator geral, também relatado na literatura (Heausler \& Thompson, 1988).

\section{Considerações Finais}

A realização de um novo estudo com o Teste de Criatividade Figural Infantil visando a análise da sua estrutura interna veio complementar estudos anteriores de evidências de validade e precisão desse instrumental. Estudos esses que visam, principalmente, sanar a lacuna existente de instrumentos voltados para a avaliação desse construto em crianças.

A partir da apresentação de um novo instrumento resultante de pesquisas feitas no país, pretende-se contribuir para a área da Avaliação Psicológica, ampliando as possibilidades de avaliação da criatividade e da realização de um processo de diagnóstico mais adequado às nossas características culturais. Esta avaliação se torna mais completa a partir do oferecimento de um modelo que avalie as características criativas não somente sob a forma de uma medida geral resultante da soma simples destas, mas considerando-se as diversas facetas que são compreendidas nesse construto, principalmente em relação à separação das características cognitivas e emocionais.

\section{Referências}

Carroll, J. B. (1985). Exploratory factor analysis: a tutorial. In D. K. Determan (Ed.), Current topics in human intelligence: Research and methodology (pp. 191-205). Worwoood NJ: Ablex.

Chase, C. I. (1985). Review of the Torrance Tests of Creative Thinking. In J. V. Mitchell Jr. (Ed.), The ninth mental measurements yearbook (pp. 1631-1632). Lincoln: University of Nebraska Press.

Chen, S. A., \& Michael, W. B. (1993). First-order and higherorder factors of creative social intelligence within Guilford's structure of intellect model: a reanalysis of a Guilford database. Educational and Psychological Measurement, 53, 619-641.

Clapham, M. M. (1998). Structure of figural forms A and B of the Torrance Tests of Creative Thinking. Educational and Psychological Measurement, 58, 275-283.

Enzmann, D. (1997). RanEigen: a program to determine the parallel analysis criterion for the number of principal components. Applied Psychological Measurement, 21, 232-232.

Field, A. (2009). Descobrindo a estatística usando SPSS. Porto Alegre: Artmed.

Goodwin, L. D., \& Leech G. N. (2006). Understanding correlation: factors that affect the size of r. The Journal of Experimental Education, 74, 251-266.

Guilford, J. P. (1960). The structure of the intellect model: its use and implications. New York: McGraw-Hill. 
Heausler, N. L., \& Thompson, B. (1988). Structure of the Torrance Tests of Creative Thinking. Educational and Psychological Measurement, 48, 463-468.

Kim, K. H. (2006). Is creativity unidimensional or multidimensional? Analyses of the Torrance Tests of Creative Thinking. Creativity Research Journal, 18, 251-259.

Kim, K. H., Cramond, B., \& Bandalos, D. L. (2006). The latent structure and measurement invariance of scores on the Torrance Tests of Creative Thinking-Figural. Educational and Psychological Measurement, 66, 459-477.

Kirton, M. J. (1976). Adaptors and innovators: A description and measure. Journal of Applied Psychology, 61, 622-629.

Marroco, J. (2003). Análise estatística com utilização do SPSS. Lisboa, Pt: Sílabo.

Moraes, M. F., \& Azevedo, I. (2009). Avaliação da criatividade como um contexto delicado: revisão de metodologias e problemáticas. Avaliação Psicológica, 8 (1), 1-15.

Nakano, T. C. (2003). Criatividade Figural: proposta de um instrumento de avaliação. Dissertação de Mestrado, Pontifícia Universidade Católica de Campinas. Campinas, S.P.

Nakano, T. C., \& Wechsler, S. M. (2006a). Teste Brasileiro de Criatividade Figural: Proposta de instrumento. Revista Interamericana de Psicologia, 40, 103-110.

Nakano, T. C., \& Wechsler, S. M. (2006b). Teste Brasileiro de Criatividade Figural: Proposta de normas. Avaliação Psicológica, 5, 159-170.

Nakano, T. C., Wechsler, S. M. \& Primi, R. (2011). Teste de Criatividade Figural Infantil: Manual técnico. São Paulo: Editora Vetor.

Plass, H., Michael, J. J., \& Michael, W.B. (1974). The factorial validity of the Torrance Tests of Creative Thinking for a sample of 111 sixth-grade children. Educational and Psychological Measurement, 34, 413-414.

Reise, S. P., Waller, N. G., \& Comrey, A. L. (2000). Factor analysis and scale revision. Psychological Assessment, 12, 287-297
Runco, M. A., \& Mraz, W. (1992). Scoring divergent thinking tests using total ideational output and a creativity index. Educational and Psychological Measurement, 52, 213-221.

Sternberg, R. J. \& Grigorenko, E. L. (2001). Guilford's structure of intellect model and model of creativity: contributions and limitations. Creativity Research Journal, 13, 309-316.

Stevens, J. P. (1992). Applied multivariate statistics for the social sciences. Hillsdale, NJ: Erlbaum.

Tabachnick, B. G., \& Fidell, L. S. (1996). Using multivariate statistics. New York: HarperCollins.

Torrance, E. P. (1966). Torrance tests of creative thinking. Lexington: Personnel Press.

Torrance, E. P., \& Ball, O. E. (1978). Streamlined scoring and norms for figural form $A$ and $B$. Athens: Georgia Studies of Creative Behavior.

Torrance, E. P., \& Ball, O. E. (1990). Streamlined scoring and interpretation guide and norms manual verbal and figural form $B$. Bensenville: Scholastic Testing Service.

Torrance, E. P., \& Safter, H. T. (1999). Making the creative leap beyond. Buffalo, NY: Creative Education Foundation.

Watkis, M. W. (2006). Determining parallel analysis criteria. Journal of Modern Statistical Methods, 5 (2), 344-346.

Wechsler, S. M. (2004). Avaliação da criatividade por figuras e palavras: testes de Torrance - versão brasileira. ( $2^{\mathrm{a}}$ ed. revisada). Campinas: Lamp / Impressão Digital do Brasil.

Recebido em 28.07.2010

Primeira decisão editorial em 02.03.2011

Aceito em 31.01.2012 